\title{
Le retour du mythe en modernité
}

Le mythe de l'État chez Cassirer et son expérience de l'exil

Le retour du mythe en modernité. Le mythe de l'État chez Cassirer et son expérience de l'exil

The Resurgent Forces of the Myth in the Modern Era. Cassirer's Myth of the State and his Experience of Exile

\section{Yves Bizeul}

\section{(2) OpenEdition \\ Journals}

\section{Édition électronique}

URL : http://journals.openedition.org/ceg/4283

DOI : $10.4000 /$ ceg.4283

ISSN : 2605-8359

\section{Éditeur}

Presses Universitaires de Provence

Édition imprimée

Date de publication : 2 mai 2019

Pagination : $37-48$

ISBN : 979-10-320-0214-8

ISSN : 0751-4239

\section{Référence électronique}

Yves Bizeul, «Le retour du mythe en modernité », Cahiers d'Études Germaniques [En ligne], 76 | 2019, mis en ligne le 02 novembre 2020, consulté le 25 janvier 2021. URL : http://journals.openedition.org/ ceg/4283 ; DOI : https://doi.org/10.4000/ceg.4283 


\section{Le retour du mythe en modernité Le mythe de l'État chez Cassirer et son expérience de l'exil}

Yves BIZEUL

Université de Rostock, Institut für Politik- und Verwaltungswissenschaften

Dans son ouvrage Die Philosophie der symbolischen Formen, Cassirer définit le mythe comme une narration qui émerge d'un travail de mimésis et se nourrit de métaphores. Il ne s'agit pas à ses yeux d'un récit infantile et illogique. Cassirer le considère au contraire, dans le sillage de la Scienza nuova de Giambattista Vico ${ }^{1}$, comme une manière de maîtriser le monde et de produire du savoir ${ }^{2}$. Ernst Blumenberg a salué en son temps cette valorisation d'un mythe qui pour Cassirer structure le monde en s'appuyant sur la distinction entre le sacré et le profane, tout en regrettant qu'il soit pour le philosophe de Hambourg une forme symbolique dépassée en modernité et non une narration qui ne passe pas ${ }^{3}$.

Le mythe mimétique relève prioritairement pour Cassirer, en tant que Denk- et Lebensform, du domaine de l'expressivité ou de l'interpellation (Ausdruck), non de la description ou de la (re)présentation (Darstellung) ou bien de la signifiance (Bedeutung) ${ }^{4}$. Il s'agit d'une forme symbolique originaire d'où découlent toutes les autres par un processus dialectique. Par forme symbolique, il faut entendre avec Jean Lassègue « ce qui rend possible la constitution d'une matière en signifiant ${ }^{5}$ ». Elle met de l'ordre dans le monde et contribue ainsi à une objectivation de l'esprit.

Cassirer souligne que le mythe est même la source de toutes les autres formes symboliques, non seulement de l'art, du langage et de la religion, mais aussi de la science et il affirme :

1. Giambattista Vico, Principi di Scienza Nuova d'intorno alla Comune Natura delle Nazioni, Naples, F. Mosca, 1725.

2. Ernst Cassirer, Die Philosophie der symbolischen Formen. Zweiter Teil. Das mythische Denken, Darmstadt, Wissenschaftliche Buchgesellschaft, 9e éd., 1994.

3. Hans Blumenberg, Arbeit am Mythos, Frankfurt a. M., Suhrkamp, 1979, p. 59. Joseph Mali, « The Myth of the State revisited. Ernst Cassirer and modern political theory », in Jeffrey Andrew Barash (dir.), The Symbolic Construction of Reality. The Legacy of Ernst Cassirer, Chicago, University of Chicago Press, 2008, p. 135-162, p. 143.

4. Cassirer, Philosophie, p. 80. Certes, Cassirer souligne que le monde des images du mythe et le langage - qui relève, lui, avant tout de la (re)présentation - forment tous deux une dimension particulière de la Darstellung (ibid., p. 56-57). Mais alors que le langage a pour fonction principale de produire du sens, l'Ausdruck est associé par Cassirer, avant tout, au rituel magique.

5. Jean Lassègue, « Note sur l'actualité de la notion de forme symbolique », in Methodos 2, 2002, L'esprit. Mind/Geist, p. 155-182, p. 162. 
Car le point de départ authentique de tout devenir de la science, l'élément immédiat qui est à son origine, se trouve moins dans la sphère sensible que dans l'intuition mythique. [...] On ne peut comprendre intégralement le développement de la science - considéré en un sens idéel et non temporel - qu'à la condition de montrer comment elle procède de la sphère de l'immédiateté mythique et s'élabore à partir d'elle ${ }^{6}$.

Il n'en reste pas moins que la science est, pour Cassirer, le résultat d'un détachement progressif de la raison de la sphère mythique ${ }^{7}$. C'est que cette dernière correspond à ses yeux à une perception du monde dépassée, principalement affectuelle et émotionnelle. Elle résulterait d'un débordement d'émotions; d'une mise en scène de drames qui découlent de l'existence de forces antinomiques. Selon Cassirer, le mythe a, en outre, le défaut de ne pas déboucher, à la différence de la religion, sur un impératif éthique.

\section{Le mythe politique moderne dans The Myth of the State}

Cassirer, le spécialiste de l'analyse des mythes, a été, lui-même, l'une des nombreuses victimes du retour violent du mythe en modernité. D'origine juive, il dut abandonner sa chaire de philosophie à l'université de Hambourg dès 1933 et s'exiler, d'abord en Grande-Bretagne, puis en Suède, dont il reçut la nationalité en 1939. En 1941, il quitta la Suède pour se rendre aux États-Unis.

Durant cette période d'exil, le focus de son intérêt pour le mythe se déplace : Cassirer se consacre désormais en priorité à l'étude des mythes politiques modernes. Dans une lettre à Susanne Langer du 18 avril 1944, il évoque un livre à paraître qui portera sur l'origine et le caractère de ces mythes et dans lequel il cherchera à répondre à la question : comment est-il possible que la pensée mythique, considérée la plupart du temps comme «primitive » et « prélogique », ait pu avoir un tel impact sur l'organisation actuelle de notre vie politique et sociale? La rédaction de l'ouvrage rédigé en anglais et portant le titre The Myth of the State est terminé en avril 1945 mais il n'a été publié qu'en 1946 de manière posthume, un an après le décès de l'auteur.

Pour Cassirer, le retour massif des narrations mythiques dans le sillage du national-socialisme est un retour du refoulé déclenché par les grandes crises sociétales et économiques des années 1920 et 1930. Elles auraient mis à mal les représentations raisonnables de la réalité et facilité la fuite d'un grand nombre d'Allemands dans le préjugé, la religion et le mythe. Depuis sa lecture des études anthropologiques de Bronisław Malinowski, Cassirer est persuadé que le mythe est le résultat de crises $^{8}$. Alors que dans les sociétés « sauvages » ces dernières prennent

6. Ernst Cassirer, Langage et mythe : à propos des noms de dieux, Paris, Éditions de Minuit, 1925, p. 11.

7. Maud Hagelstein, Origine et survivances des symboles. Warburg, Cassirer, Panofsky, Hildesheim, Georg Olms Verlag, 2014, p. 173-175.

8. Bronisław Malinowski, The Foundations of Faith and Morals. An Anthropological Analysis of Primitive Beliefs and Conduct with Special Reference to the Fundamental Problems of Religion and Ethics, London, Oxford University Press, H. Milford, 1936. 
la forme de phénomènes naturels, elles sont de nature économique et sociale dans les sociétés modernes.

Au début du Myth of the State, on trouve un premier chapitre consacré à la définition du mythe. Cassirer le conçoit, dans la ligne de ses analyses plus anciennes, comme une « émotion qui prend la forme d'une image " ${ }^{9}$. Dans une seconde partie, il décrit la longue histoire de la lutte entre le mythos et le logos. Le mythe est pour lui un mode de vie (Lebensform) émergeant d'une «conscience mythique » qui inclue également la magie, la religion, la croyance et les rituels. Il incarne la dimension affectuelle de la politique et a été instrumentalisé, à l'aube des temps modernes, par les adeptes de la théologie politique au cours de la lutte qui les opposa aux partisans du droit naturel.

Cassirer voit les germes du retour du mythe en modernité avant tout dans la philosophie de Hegel et dans les écrits des Romantiques allemands qui prônèrent la prééminence du tout organique sur la logique et les sciences ${ }^{10}$. Il présente Hegel, Carlyle et Gobineau comme des auteurs ayant indirectement contribué par leurs écrits au triomphe du national-socialisme : Carlyle par son culte du héros, Gobineau par sa doctrine racialiste et Hegel par sa théorie de l'État. On trouve également dans le livre de Cassirer des analyses très critiques de "l'astrologie de l'histoire » d'Oswald Spengler ainsi que de la philosophie existentialiste de Martin Heidegger.

C'est seulement dans le dernier chapitre de l'ouvrage que le mythe politique moderne proprement dit est abordé. Cette partie ne prend dans l'ensemble du livre qu'une place restreinte, limitée à une vingtaine de pages. De plus, Cassirer ne s'intéresse ici qu'au seul mythe national-socialiste de l'État. The Myth of the State est un livre engagé devant contribuer à déconstruire les narrations mythiques d'Alfred Rosenberg et de son ouvrage Der Mythus des 20. Jahrhunderts ${ }^{11}$.

Il veut rendre compte de l'origine, de la structure et de la technique des mythes politiques modernes afin de mieux combattre les nationaux-socialistes sur leur propre terrain, celui du symbolique, et il affirme à la fin de son ouvrage : " Il nous faut regarder l'adversaire droit dans les yeux de manière à comprendre comment on peut lutter contre lui ${ }^{12} »$. Dans le même temps, il est conscient des limites de son entreprise. Il précise : «Détruire les mythes politiques est hors de portée du pouvoir de la philosophie. Un mythe est en quelque sorte invulnérable. L'argument rationnel perle sur lui et il ne se laisse pas réfuter par des syllogismes ${ }^{13} »$.

9. Traduit de l'anglais par Y. B. « emotion turned into an image ». Ernst Cassirer, The Myth of the State, New Haven, Yale University Press, 1946, p. 45-60.

10. Theo Stammen, « Ernst Cassirers Kritik der politischen Romantik », in Wolfgang Leidhold (dir.), Politik und Politeia. Formen und Probleme politischer Ordnung. Festgabe für Jürgen Gebhardt zum 65. Geburtstag, Würzburg, Königshausen \& Neumann, 2000, p. 219-220.

11. Fabien Capeillières, « Cassirer penseur politique. The Myth of the State contre Der Mythus des 20. Jahrhunderts », in Cahiers de Philosophie politique et juridique 24, 1994, p. 175-204.

12. "We should see the adversary face to face in order to know how to combat him », Cassirer, The Myth of the State, p. 296.

13. «It is beyond the power of philosophy to destroy the political myths. A myth is in a sense invulnerable. It is impervious to rational argument; it cannot be refuted by syllogisms ", ibid. 
L'analyse cassirienne purement négative du mythe fait sens et est légitime dans le contexte de l'époque : l'impératif d'un engagement politique rendait alors difficile toute neutralité axiologique. Cassirer voulait immuniser, dans son exil américain, les démocrates de ce pays contre les dangers du totalitarisme. Il s'agissait de préserver les acquis des Lumières contre un obscurantisme qui menaçait de gagner non seulement l'ensemble de l'Europe, mais aussi le monde entier.

Pellegrino Favuzzi relève, en s'appuyant sur le Myth of the State mais aussi sur d'autres écrits de Cassirer de l'époque, que ce dernier établit, dans ses analyses, une distinction entre quatre modes de propagande des nationaux-socialistes. Ils utilisent, en premier lieu, une forme langagière rudimentaire de manière à susciter des émotions fortes comme la haine, la peur, la colère, l'arrogance ou le mépris. Ils organisent, en second lieu, de grands rituels collectifs afin de dérober à l'individu son autonomie et son sens de la responsabilité individuelle. Celui-ci devient alors un instrument malléable dans les mains du parti. Même les plus érudits et les plus intelligents se transforment en marionnettes. En troisième lieu, le Führer incarne, en tant que personnage mythique, l'ordre sociétal à rétablir. Il se comporte en magicien et canalise les tensions émotionnelles en les concentrant sur un point nodal. En quatrième lieu, les nationaux-socialistes ont réintroduit la figure de l'ennemi mortel qui est au centre de tout drame, et plus encore de tout récit mythique. Pour Cassirer, les juifs sont leur cible privilégiée dans la mesure où leur religion les conduit à critiquer le mythe et à mettre en exergue la dimension éthique de l'existence ${ }^{14}$.

\section{Une version amendée du Myth of the State?}

La philosophe Chiara Bottici est convaincue que cette attitude militante ne pouvait conduire qu'à une impasse dans la mesure où la pauvreté de l'analyse cassirienne du mythe politique moderne, réduit à un simple instrument de pouvoir, à une technique de domination, n'est guère, selon elle, en mesure de livrer un contrepoison efficace au totalitarisme. Elle reproche à Cassirer, dans son commentaire du Myth of the State $^{15}$, de ne voir dans le mythe qu'un instrument élaboré par des « sauvages » ou des nationaux-socialistes, donc, dans les deux cas, par des penseurs primitifs, et elle reprend à son compte la critique de Hans Blumenberg qui regrettait que le mythe ne soit, aux yeux de Cassirer, qu'un terminus ad quem, autrement dit le fruit d'une régression, et non un terminus post quem ${ }^{16}$. Bottici évoque également la critique d'Eric Voegelin adressée à l'époque à Cassirer. Voegelin lui reproche, dans une recension du Myth of the State parue en $1947^{17}$, d'avoir esquissé, sans le dire

14. Pellegrino Favuzzi, « Kulturphilosophie und Politischer Mythos. Ernst Cassirers Kritik der mythischpolitischen Vernunft », in Zeitschrift für Politik 62, 2015, p. 421-441, p. 437.

15. Chiara Bottici, «Wer hat Angst vor Cassirers "The Myth of the State”? Elemente eines philosophischen Krimis », in Yves Bizeul, Stephanie Wodianka (dir.), Mythos und Tabula rasa. Narrationen und Denkformen der totalen Auslöschung und des absoluten Neuanfangs, Bielefeld, transcript Verlag, 2018.

16. Blumenberg, Arbeit am Mythos, Frankfurt a. M., Suhrkamp, 1979, p. 186.

17. Eric Voegelin, « The Myth of the State, By Ernst Cassirer », in The Journal of Politics 9, 1947, p. 445-447. 
ouvertement, une philosophie de l'histoire; cette dernière étant censée progresser vers le dépassement de l'irrationalité primitive grâce à une rationalité grandissante. Bottici évoque enfin une interview de Cassirer portant sur ses travaux récents, parue en 1944 dans la revue Fortune et dans laquelle elle décèle un fort relent scientiste et comtien ${ }^{18}$. Mais elle fait part également de son étonnement lorsqu'elle découvrit une étude plus fine du mythe politique dans un manuscrit de Cassirer moins connu. Il se trouve dans le tome 9 des Nachgelassene Manuskripte und Texte édités par John Michael Krois et Christian Möckel et est intitulé : The Myth oft the State. Its Origin and its Meaning. Part III : The Myth of the Twentieth Century ${ }^{19}$.

Ce manuscrit, qui contient nombre d'analyses identiques à celles du Myth of the State, commence par l'affirmation selon laquelle l'homme n'est pas uniquement un animal rationnel comme l'affirme Aristote, mais également un animal producteur de mythes, et qu'il ne peut que très partiellement dépasser cette condition première ${ }^{20}$. Cassirer souligne dans ce texte que l'histoire n'a jamais été entièrement dépourvue d'éléments mythiques, ces derniers étant un composant indissoluble de la nature humaine et ne pouvant être complètement éradiqués. Il soutient que

[1']organisme de la culture humaine ne triomphe pas par la disjonction et le rejet des éléments mythiques. Mais il génère de nouveaux et constructifs pouvoirs de la pensée, des forces éthiques neuves, des énergies créatives d'imagination artistique nouvelles permettant de contrebalancer ces éléments. Il est incontestable que l'équilibre qui est ainsi réalisé entre des forces variées et antagonistes n'est jamais acquis. Il ne s'agit pas d'un équilibre stable, mais labile. Il n'est pas fermement assuré, mais livré à toutes sortes de dérèglements ${ }^{21}$.

\section{Il poursuit par une vision quasi apocalyptique du monde politique :}

Le mythe a été la première force ayant permis de donner une forme et d'organiser la société humaine. C'est dans ce domaine qu'il a ses racines inextirpables les plus profondes. Dans les temps sereins et pacifiques, dans les périodes de relative stabilité et de sécurité, il paraît aisé de contrôler et de contrebalancer les forces mythiques occultées. Mais elles ne sont, en réalité, ni défaites, ni domptées. Toute crise dans notre vie sociale peut conduire à leur réactivation soudaine. La politique est, non seulement au plan pratique, sociologique et économique, mais aussi dans ses fondations théoriques, la chose la plus incertaine et la plus précaire qui soit. Dans ce domaine, nous n'évoluons jamais sur un fondement ferme et stable. La politique reste toujours

18. Ernst Cassirer, « The Myth of the State », in Fortune 29, 1944, p. 165-169.

19. Cassirer, The Myth of the State. Its Origin.

20. Traduit de l'anglais par Y. B. « L'homme n'est pas simplement un animal rationnel, il est et demeure un animal producteur de mythes. Le mythe est inévitablement le propre de l'homme. Il est une partie et une parcelle de la nature humaine. Nous pouvons appliquer ici la devise "Naturam expelles furca, tamen usque recurret" ». « Man is not only an "animal rationale", a rational animal; he is and remains a mythical animal. Myth inevitably belongs to man; it is part and parcel of human nature. We may apply to it the device "Naturam expelles furca, tamen usque recurret" ", ibid., p. 172.

21. Traduit de l'anglais par Y. B. «[t]he organism of human culture does not succeed in separating and excreting the mythical elements. But it develops new and constructive powers of thought, new ethical forces, new creative energies of artistic imagination to counterpoise these elements. It is true that the equilibrium, which is attained here between various and antagonistic forces, is never an accomplished fact. It is not a static but a labile equipoise; it is not firmly established but liable to all sorts of disturbance. », Ernst Cassirer, « The myth of the state. Its origin and its meaning. Third part: the myth of the twentieth century "), in Cassirer, Nachgelassene Manuskripte und Texte: $\mathrm{Zu}$ Philosophie und Politik mit Beilagen, éd. par John Michael Krois, Christian Möckel, t. 9, Hamburg, Felix Meiner Verlag, 2008, p. 167-224, p. 180. 
"instabilis tellus, innabilis unda". Nous restons sur un terrain volcanique et nous devons nous préparer à des convulsions et éruptions soudaines. Face au défoulement d'émotions politiques violentes, la voix de la pensée rationnelle est trop faible pour résonner fermement et se faire entendre. Dans ces moments, la pensée mythique reprend toute sa vigueur. Elle retrouve toute sa place et est réintronisée. Elle revendique et usurpe tous ses anciens droits. [...] Elle était restée tapie à l'arrière-plan, attendant son heure et sa chance. Cette heure sonne lorsque toutes les autres forces de cohésion de la vie sociale se dissolvent, lorsqu'elles ne sont plus assez vigoureuses pour pouvoir brider les pouvoirs mythiques démoniaques et les contrôler ${ }^{22}$.

Dans le Nachlass le mythe est également décrit comme un instrument qui peut être mis au service de la rationalité scientifique. Bottici voit ici un parallèle avec la thèse principale de l'ouvrage de Horkheimer et d'Adorno La Dialectique des Lumières. Les crimes nationaux-socialistes ont été rationnellement et scientifiquement organisés. Nos mythes modernes ne sont pas mystiques, mais au contraire le résultat d'un travail analytique, et sont porteurs d'une forme de rationalité propre. C'est le cas du mythe de la race étudié par Cassirer et qui a été le fruit d'une longue élaboration théorique par des auteurs comme Gobineau, Chamberlain ou Rosenberg. Les mythes sont désormais fabriqués en plein jour et de manière systématique.

En outre, comme le fait remarquer Bottici, la version du Nachlass contient des analyses du national-socialisme, de la République de Weimar et du marxisme qui donnent à ce texte une pertinence politique particulière. Cassirer y insiste sur le fait que la force de persuasion du mythe du $\mathrm{XX}^{\mathrm{e}}$ siècle résulte de son statut de croyance quasi-religieuse. Au fondement de la religion et du mythe on trouve un « désir collectif personnifié » comme l'affirmait l'anthropologue Edmond Doutté ${ }^{3}$. Le désir premier des Allemands, au cours de la République de Weimar, avait été le démantèlement du traité de Versailles. L'impossibilité de réaliser cet objectif dans les faits ne fit que l'attiser encore. Comme le mythe, pour Cassirer, se caractérise par sa démesure, ce désir aurait pris la forme d'une volonté de dominer le monde entier.

La plupart du temps, le mythe n'a pas d'auteur. Ce n'est toutefois pas le cas, si l'on en croit Cassirer, dans les systèmes totalitaires. Le mythe y est élaboré, préciset-il, par des artisans habiles et malins ${ }^{24}$, et le manipulateur qui a recours à cette arme est à la fois un «homo magus » et un «homo faber». Cassirer évoque le fait

22. Traduit de l'anglais par Y. B. « Myth was the first power to form and organize human society. In this sphere it has its deepest and ineradicable roots. In quiet and peaceful times, in periods of relative stability and security, it seems to be easy to check and counterbalance the hidden mythical motives. But they are not really defeated and subdued. Every crisis in our social life may lead to a sudden rebellion. Not only in its practical, in its sociological or economic conditions, but also in its theoretical foundations politics is the most insecure and precarious thing. Here we are never living on a firm and stable ground. Politics always remains "instabilis tellus, innabilis unda". We are standing on a volcanic soil and must be prepared for abrupt convulsions and eruptions. In the turmoil of violent political emotions the voice of rational thought is too feeble to make itself heard and understood. At these moments mythical thought regains its full strength. It is reinstated and enthroned; it claims and usurps all its former rights. It has never abandoned these rights; it was always lurking in the background waiting for its hour and its opportunity. This hour comes if all the other binding forces of social life seem to be dissolved; if they are no longer strong enough to bridle the demonic mythical power[s] and bring them under control. », ibid.

23. Edmond Doutté, Magie et religion dans l'Afrique du Nord, Alger, Adolphe Jourdan, 1908, p. 601.

24. « made by very skilful and cunning artisans », Cassirer, The Myth of the State. Its Origin, p. 200. 
que ce type de mythe est identique à une arme militaire moderne, un avion ou une mitrailleuse.

Dans le Nachlass, il affirme, en outre, que les marxistes, en mettant l'accent trop unilatéralement sur la lutte des classes, n'ont pas été à même de comprendre la vraie nature du national-socialisme, la puissance du mythe étant pour eux une «terra incognita $»^{25}$. Les nationaux-socialistes étaient, affirme-t-il, plus habiles que les communistes. Ils ont d'abord consacré leurs forces à un réarmement spirituel avant de les investir dans le réarmement matériel. Avec Antonio Gramsci on peut affirmer qu'ils ont d'abord conquis l'hégémonie culturelle, une hégémonie nécessaire à toute prise de pouvoir politique.

Les nationaux-socialistes ont déclaré la vérité scientifique scélérate et ont combattu la méthode historico-critique. Les faits historiques ont perdu ainsi de leur pertinence. On assista au règne des fake news. Cassirer relève également que la religion et l'éthique ont été des forteresses qui ont su résister à l'assaut du mythe. Dans ce cas, la tactique des nationaux-socialistes fut de les remodeler de l'intérieur, en injectant leur propre idéologie comme un poison et en pervertissant les principes de la religion et de l'éthique. Religion et éthique ont été ainsi dépossédées de leur vérité et de leurs fins propres.

Le mythe ne s'évanouit donc pas en modernité; il se transforme. Alors que les références à la théorie hobbesienne de l'État moderne conçu comme « Dieu mortel » sont absentes du Myth of the State, le Cassirer du Nachlass prétend que Hobbes nous invite à déposer sur l'autel de la nouvelle divinité intramondaine étatique, non seulement nos biens et nos existences, mais également nos sentiments, nos pensées, notre morale et nos opinions religieuses. Ce n'est possible que sur le soubassement d'une pensée mythique. Alors que le Hobbes du Myth of the State met un terme, en tant que théoricien de la loi naturelle, à la pensée mythique, celui du Nachlass élabore, lui, une théologie politique de l'État.

On doit, dès lors, se poser la question de savoir si le nouveau mythe de l'État est bien l'aboutissement de la tradition de pensée allemande comme l'affirme Cassirer dans The Myth of the State, ou bien s'il n'est pas plutôt l'aboutissement de la conception absolue de la souveraineté de l'État défendue par Hobbes et par ses épigones, comme Cassirer semble le suggérer dans le Nachlass. Si c'est le cas, on peut voir dans Cassirer avec Bottici un précurseur du philosophe italien Giorgio Agamben.

À la fin du manuscrit du Nachlass on trouve quelques réflexions sur l'Éthique de Spinoza. Pour Spinoza, un argument rationnel ne peut avoir raison d'un affect. Seul un affect plus fort peut l'emporter sur un autre. Cassirer tire de cette affirmation la leçon qu'un mythe ne peut être remplacé que par un autre récit mythique. C'est ainsi que Hobbes a remplacé l'ancien Dieu mythique par le mythe de l'État moderne. La question se pose de savoir par quel mythe la narration mythique national-socialiste pourrait-elle, à l'avenir, être supplantée?

25. Ibid., p. 201. 
Eric Voegelin partageait la même conviction que Spinoza. Dans sa recension du Myth of the State, il souligne que chercher à manipuler un mythe est une entreprise dangereuse aussi longtemps que l'on ne dispose pas d'un mythe alternatif qui pourrait prendre sa place. Vaincre un mythe à l'aide de la raison peut conduire à la victoire d'un autre mythe, aujourd'hui inconnu, mais qui peut être tout aussi, ou encore plus, dangereux que le premier. Voegelin ne semble pas connaître la version du Nachlass, qui abonde dans son sens.

Pour Bottici, le Myth of the State aurait gagné en pertinence si la version du Nachlass avait été reprise en l'état dans l'ouvrage définitif. Elle parle, de manière imagée, d'un « meurtre » qui aurait eu lieu lors de la publication du Myth of the State, les réflexions sur l'existence du mythe à toutes les époques et dans toutes les sociétés ayant été oblitérées dans la version finale de l'ouvrage.

Bottici se met en quête des « meurtriers » de la version du Nachlass. Le suspect numéro un est, selon elle, Charles Hendel, qui fut responsable, après la disparition de Cassirer, du travail d'édition du Myth of the State. Hendel relève que Cassirer n'a pas donné son autorisation personnelle à la publication de la troisième partie du livre et il évoque deux complices possibles du soi-disant «meurtre» : le professeur Brand Blanshard et le docteur Friedrich Lenz. Par ce « crime » on aurait voulu préserver la priorité accordée autrefois par Cassirer à la science sur le mythe. L'affirmation selon laquelle le national-socialisme serait un produit de la science moderne et rationnelle aurait pu obscurcir l'image du philosophe néo-kantien attaché à la tradition des Lumières.

Mais Bottici évoque encore d'autres possibilités. Il se pourrait que l'on soit en présence de deux textes différents rédigés par Cassirer lui-même. Un autre scénario est que l'on ait affaire à une sorte de « suicide » : ce serait Cassirer lui-même qui aurait édulcoré son texte de manière à le rendre conforme à l'image qu'on avait de lui aux États-Unis. Il aurait craint les conséquences pour sa notoriété d'une thèse trop provocatrice, selon laquelle on trouverait, au plus profond de la logique de la modernité, le mythe.

\section{Les limites de l'analyse de Bottici}

Pour Bottici, l'analyse cassirérienne du mythe dans Die Philosophie der symbolischen Formen et le Myth of the State serait déficitaire et rudimentaire. Cette opinion n'est pas partagée par tous les spécialistes du philosophe de Hambourg. La question se pose de savoir si pour ce dernier, les trois fonctions des formes symboliques évoquées plus haut ont la même valeur ou bien si l'histoire est comprise par ce dernier comme un crescendo allant de l'expressivité à la signification, ce qui correspondrait aux thèmes des trois tomes de l'ouvrage (le langage, la pensée mythique et la phénoménologie de la connaissance) ainsi qu'à la thèse de Bottici. Cette dernière interprétation, répandue dans la littérature sur l'auteur ${ }^{26}$, est concordante avec le fait que Cassirer

26. John Michael Krois, Cassirer - Symbolic Forms and History, New Haven, Yale University Press, 1987; Krois, « Problematik, Eigenart und Aktualität der Cassirerschen Philosophie der symbolischen 
a été un néo-kantien et un adepte des Lumières auxquelles il a consacré un ouvrage important.

Le plus souvent, toutefois, les trois fonctions ne sont pas placées par Cassirer dans un rapport chronologique, et encore moins hiérarchique ${ }^{27}$. C'est ainsi que le langage est à ses yeux un mixte d'expressivité, de représentation et de signification et qu'il est étroitement lié au mythe, même si les deux diffèrent dans leur structure respective. Cassirer affirme :

De ce point de vue, le mythe, de même que l'art, le langage et la connaissance sont des symboles : non pas en ce sens qu'ils désigneraient une réalité préexistante sous la forme de l'image, de l'allégorie qui indique ou interprète, mais dans la mesure où chacun d'eux crée un univers de sens à partir de lui-même ${ }^{28}$.

Bottici met l'accent de manière trop unilatérale sur la téléologie et la phénoménologie de type hégélien qui se dissimuleraient derrière les réflexions de Cassirer sur le mythe. Elle refuse même de suivre Joseph Mali qui voit une inflexion des thèses de Cassirer sur le mythe entre Die Philosophie der symbolischen Formen et le Myth of the State. Mali relève que si le philosophe de Hambourg a bel et bien été incapable de reconnaître toute la valeur du mythe pour la construction des communautés politiques dans Die Philosophie der symbolischen Formen, il a souligné lui-même avoir voulu infléchir par la suite ses anciennes analyses, qui étaient largement répandues parmi les intellectuels libéraux de son époque ${ }^{29}$.

Pour Bottici, on ne trouverait une véritable inflexion de la pensée de Cassirer que dans le manuscrit du Nachlass. Nous avons vu ses arguments, mais les différences évoquées entre le texte du Nachlass et l'ouvrage final ne nous paraissent pas aussi décisives qu'elle le prétend dans le but de donner un plus grand impact à sa propre interprétation. Dans les deux textes, le mythe est contraire à la raison et constitue une menace pour la modernité. Cassirer ne prend pas en compte les fonctions positives du mythe politique.

En outre, l'ambiguïté repérée par Bottici est déjà présente chez le Cassirer de Die Philosophie der symbolischen Formen. Leopoldo Iribarren évoque « deux directions théoriques virtuellement contradictoires entre elles » qui traversent la pensée cassirérienne dès la période dite de Hambourg :

On trouve là, d'une part, un élargissement important de la théorie de la connaissance - qui dans l'approche néokantienne de l'école de Marbourg concernait prioritairement les sciences mathématiques et physique - vers la totalité des sphères de l'activité humaine. De ce fait, la théorie de la connaissance, qui fut le premier grand thème philosophique de Cassirer, se trouve

Formen », in Hans-Jürg Braun, Helmut Holzhey, Ernst Wolfgang Orth (dir.), Über Ernst Cassirers Philosophie der symbolischen Formen, Frankfurt a. M., Suhrkamp, 1988, p. 15-44. La question de l'impact de la téléologie néokantienne sur la pensée du mythe de Cassirer est posée par Markus Tomberg dans son ouvrage : Der Begriff von Mythos und Wissenschaft bei Ernst Cassirer und Kurt Hübner, Münster, Lit Verlag, 1996. Tomberg ne donne pas de réponse claire sur ce point.

27. Esther Oluffa Pedersen, Die Mythosphilosophie Ernst Cassirers: zur Bedeutung des Mythos in der Auseinandersetzung mit der Kantischen Erkenntnistheorie und in der Sphäre der modernen Politik, Würzburg, Königshausen \& Neumann, 2009.

28. Cassirer, Langage et mythe, p. 16.

29. Mali, The Myth of the State Revisited. 
refondue en une théorie générale de la culture, où le terme de culture ne désigne pas une totalité donnée, mais l'ensemble de relations entre des orientations symboliques diverses et parfois opposées où l'esprit est simultanément objectivé. Mais, d'autre part, Cassirer ne cherche pas moins, dans la perspective de ce qu'il appelle une phénoménologie de la culture, à élucider la logique qui préside à la différenciation des mondes symboliques et à retracer la dynamique de leur succession historique commandée par la finalité cognitive ${ }^{30}$.

Cette tentative ne pouvait conduire qu'à une impasse, et Cassirer dans la dépression, comme l'atteste le passage suivant du Nachlass :

Dans la sphère politique, nous avons été, pour ainsi dire, projetés dans le chaos originel. La pensée mythique a gagné une nouvelle victoire sans précédent. Et cela ne peut rester un phénomène isolé. Dans nos théories modernes de l'État, qui ont été développées depuis le commencement $\mathrm{du} \mathrm{XX}^{\mathrm{e}}$ siècle, le mythe n'a pas simplement envahi un espace particulier, il a conquis toute la civilisation humaine. Toutes ces théories maintiennent et défendent une conception "totalitaire" de 1'État ${ }^{31}$.

Pour Cassirer, le mythe est, en modernité, avant tout un récit « pathologique ». Sa situation d'exilé dans un monde civilisé rétréci comme peau de chagrin le conduit à adopter cette position pessimiste.

\section{Épilogue}

La narration mythique a, selon Cassirer, une fonction interpellative. Roland Barthes parle à ce propos d'adhomination, de récit ad hominem ${ }^{32}$, et Hans Blumenberg d'insister sur le fait que le mythe ne produit pas que du sens (Sinn), ce qui est le propre de toute narration, mais aussi et surtout de la significativité (Bedeutsamkeit), ${ }^{33}$ un terme emprunté à Wilhelm Dilthey et à Erich Rothacker et qui place d'emblée le mythe dans l'ordre de la performativité. Le mythe nous interpelle directement et existentiellement en raison de sa pertinence pour notre existence individuelle et pour celle de notre communauté. On ne doit pas chercher à l'évaluer à l'aune du vrai et du faux, mais s'interroger sur le point de savoir s'il nous parle (ou s'il parle à ceux auxquels il est adressé) ou non.

Si les mythes politiques sont moins complexes que les mythes archaïques et religieux, dont ils reprennent certains éléments, ils ont souvent une structure composite intégrant non seulement plusieurs mythèmes, mais aussi plusieurs narrations, comme le montre l'étude de Rolf Reichardt sur le mythe de la prise de la

30. Leopoldo Iribarren, « Langage, mythe et philologie dans la Philosophie des formes symboliques d'Ernst Cassirer », in Revue germanique internationale 15, 2012, p. 95-114, p. 97.

31. Traduit de l'anglais par Y. B. «In the political sphere we were, so to speak, thrown back to the original chaos. Mythical thought won a new and unprecedented victory. And this could not remain an isolated phenomenon. In our modern theories of the state, developed since the beginning of the twentieth century, myth has not only invaded a special province; it has conquered the whole of human civilisation. All these theories maintain and defend a "totalitarian" conception of the state. », Cassirer, The Myth of the State. Its Origin, p. 173.

32. Roland Barthes, «Le mythe, aujourd'hui », in Mythologies, Paris, Éditions du Seuil, 1957, p. 181-233.

33. Blumenberg, Arbeit am Mythos, p. 68-126. 
Bastille $^{34}$. Ce dernier comprend un mythe pivot de fondation républicaine, un mythe de menace et de conspiration, un mythe de rédemption et d'actes héroïques, un mythe de réconciliation et un «mythe missionnaire parareligieux de supériorité ».

Alors que les mythes des sociétés sauvages ne divisent pas, dans la mesure où ils ne connaissent ni doctrines, ni dogmes et que le monde n'y est pas présenté comme le lieu d'un conflit entre des forces antagonistes (entre le bien et le mal, entre les frères dans la foi et les hérétiques ou entre les prolétaires et les capitalistes) mais comme un tout indivisible qui peut connaître des périodes de chaos, des drames et qui doit être ordonné ${ }^{35}$, les mythes politiques, eux, ont une structure toute autre. Instrumentalisés, si ce n'est façonnés par les grandes idéologies et religions séculaires du $\mathrm{xx}^{\mathrm{e}}$ siècle, comme le relève Ernst Cassirer, le mythe est mis au service d'une croyance politique. La narration mythique peut alors être contenue dans une image ou une photo ou bien même se résumer à un simple slogan politique comme dans le cas du mythe de la grève générale évoqué par George Sorel.

L'instrumentalisation idéologique du récit mythique n'est possible qu'en raison de ce que Hans Blumenberg a appelé l' " ouverture » principielle du mythe. Cette « ouverture» est utilisée dans les régimes totalitaires pour promouvoir des oppositions dichotomiques. Le mal ou l'impur doit être éliminé, si nécessaire par la lutte armée ou l'utilisation de la violence, la plupart du temps par une bataille décisive ou par une insurrection, ce qui explique la structure hautement dramatique de tels récits. Karl Marx, dans une lettre de 1871 adressée au médecin et révolutionnaire Ludwig Kugelmann, désignait les communards comme des caeloclastes, des conquérants du ciel (Himmelsstürmer), un terme issu à l'origine de l'eschatologie chrétienne. Les bolcheviques désignèrent de manière plus prosaïque, mais non moins symbolique, leur insurrection comme « l'assaut du Palais d'Hiver ».

Le mythe perd sa «liberalité » (Blumenberg) lorsqu'il est mis au service d'idéologies politiques modernes de « suture ${ }^{36}$. René Kaës comprend sous ce terme un discours idéologique qui prend la forme d'un objet de fétichisme ${ }^{37}$. C'est le cas de la plupart des mythes politiques de la " première modernité » (Ulrich Beck) qui portent en eux la structure du « soit/ soit » propre aux grandes idéologies politiques (nationalisme, communisme, national-socialisme).

34. Rolf Reichardt, « Die Stiftung von Frankreichs nationaler Identität durch die Selbstmystifizierung der Französischen Revolution am Beispiel der "Bastille" ", in Helmut Berding (éd.), Mythos und Nation. Studien zur Entwicklung des kollektiven Bewußtseins in der Neuzeit 3, Frankfurt a. M., Suhrkamp, 1996, p. 133-163.

35. On trouve certes dans les mythes archaïques des codes binaires, comme l'a montré Lévi-Strauss, mais ces oppositions ne sont pas structurelles. Elles sont levées en partie par des figures ou des concepts transversaux qui forment autant de ponts entre la nature et la culture, le ciel et la terre, le cru et le cuit ou la vie et la mort. On peut évoquer ici le rôle joué par la figure du trickster ou « décepteur » dans les mythes amérindiens analysés par Lévi-Strauss.

36. Yves Bizeul, " Politische Mythen im Zeitalter der "Globalisierung" », in Klaudia Knabel, Dietmar Rieger, Stephanie Wodianka (éds.), Nationale Mythen - kollektive Symbole. Funktionen, Konstruktionen und Medien der Erinnerung, Göttingen, Vandenhoeck \& Ruprecht, 2005, p. 17-36, p. 36.

37. René Kaës, L'Idéologie, études psychanalytiques : mentalité de l'idéal et esprit du corps, Paris, Dunod, 1980, p. 225-227. 
Même dans notre « seconde modernité », où prévaut selon Beck non plus le paradigme du « soit/ soit » mais celui du « aussi bien que », un certain nombre de mythes politiques continuent à porter en eux la structure dichotomique des idéologies de clôture qui les engendre ${ }^{38}$ (Claviez 1998). C'est le cas du mythe nationaliste de Jeanne d'Arc, instrumentalisé par le Front National lors de la fête du $1^{\mathrm{er}}$ mai. Dans le même temps, le retour actuel du religieux fondamentaliste et du nationalisme conduit à une renaissance des mythes politiques y afférant. Dans le contexte présent, la pensée du mythe du théoricien de la culture et des formes symboliques Cassirer, en dépit de ses limites, nous parle encore, à nous qui sommes confrontés à un populisme de droite qui utilise à nouveau systématiquement le mythe à des fins de pouvoir. C'est ce que nous rappelle aujourd'hui le discours trumpiste et le concept de "réalité alternative".

38. Thomas Claviez, Grenzfälle. Mythos - Ideologie, American Studies, Trier, Wissenschaftlicher Verlag Trier, 1998, p. 173-179. 